\title{
Bounds on cosmic ray-boosted dark matter in simplified models and its corresponding neutrino-floor
}

\author{
James B. Dent, ${ }^{1,2}$ Bhaskar Dutta, ${ }^{3}$ Jayden L. Newstead ${ }^{4,5,6}$ and Ian M. Shoemaker ${ }^{7}$ \\ ${ }^{1}$ Department of Physics, Sam Houston State University, Huntsville, Texas 77341, USA \\ ${ }^{2}$ Kavli Institute for Theoretical Physics, University of California, \\ Santa Barbara, California 93106-4030, USA \\ ${ }^{3}$ Mitchell Institute for Fundamental Physics and Astronomy, Department of Physics and Astronomy, \\ Texas A\&M University, College Station, Texas 77845, USA \\ ${ }^{4}$ Department of Physics, Arizona State University, Tempe, Arizona 85287, USA \\ ${ }^{5}$ Department of Physics and Astronomy, Purdue University, West Lafayette, Indiana 47907, USA \\ ${ }^{6}$ School of Physics, The University of Melbourne, Victoria 3010, Australia \\ ${ }^{7}$ Center for Neutrino Physics, Department of Physics, Virginia Tech University, \\ Blacksburg, Virginia 24601, USA
}

(Received 30 July 2019; revised manuscript received 15 May 2020; accepted 1 June 2020; published 15 June 2020)

\begin{abstract}
We study direct detection bounds on cosmic ray-upscattered dark matter in simplified models including light mediators. We find that the energy dependence in the scattering cross section is significant, and produces stronger bounds than previously found (which assumed constant cross sections) by many orders of magnitude at low dark matter mass. Finally, we compute the "neutrino-floor" that will limit future direct detection searches for cosmic ray-upscattered dark matter. While we focus on vector interactions for illustration, we emphasize that the energy dependence is critical in determining accurate bounds on any particle physics model of dark matter-cosmic ray interactions from experimental data on this scenario.
\end{abstract}

DOI: 10.1103/PhysRevD.101.116007

\section{INTRODUCTION}

The existence of a nonluminous class of matter dubbed dark matter (DM) has been firmly established, albeit only on the basis of its gravitational impact on visible matter. Further, the particle nature of this DM is unknown. A nongravitational detection of DM would provide an enormous first step in understanding its particle nature.

One of the most promising experimental avenues is to search for the small energy depositions from DM elastically scattering in very sensitive detectors on Earth [1]. This "direct detection" of DM can proceed from scattering on nuclei [2-11] or electrons [12,13]. In either case, the same interactions with ordinary matter allow high energy cosmic rays (CRs) to scatter on background DM. This has two important phenomenological impacts with observable consequences: (1) it can lead to additional CR energy losses [14], and (2) can improve detection prospects for light DM by giving such particles much larger energies so that they are more easily detected via terrestrial nuclear $[15,16]$ or

Published by the American Physical Society under the terms of the Creative Commons Attribution 4.0 International license. Further distribution of this work must maintain attribution to the author(s) and the published article's title, journal citation, and DOI. Funded by SCOAP. electron scattering [16,17]. Other recent methods for improving the bounds on light DM provide complementary probes using e.g., the Migdal effect [2,18-22], bremsstrahlung [23], cosmic microwave background (CMB) distortions [24-26], and inelastic CR collision with the atmosphere producing energetic light dark matter via meson decays [27]. We note as well that relativistic scattering at DM direct detection experiments was previously considered in the context of models in which the products of DM annihilation scatter with nuclei [28].

The existing literature on DM-CR interactions has assumed cross sections that are energy independent. This turns out to be a common occurrence for nonrelativistic $\mathrm{DM}$, but often not the case more generally. More typically, in elementary particle physics models in which the CR-DM interaction proceeds via the exchange of some new scalar or vector particle, the relativistic differential cross section depends non-trivially on the energy and mass of the participants. Moreover, DM-CR interactions probe large cross sections and can have large momentum transfers. If these large cross sections are generated by a mediating particle with mass $m_{\phi}^{2} \lesssim q^{2}$, where $q$ is the exchanged momentum, one must retain the full propagator term in the cross section.

Here we formulate the DM-CR interactions within the context of a simplified model of DM-nucleon interactions 
(e.g., [29-34]). This allows us to remain agnostic of the detailed UV-physics, yet still capture the relativistic behavior of the scattering. This formalism is commonly applied in dark matter searches using collider [35,36], indirect detection [37] and direct detection approaches [38]. We will consider simplified models of a light vector, $V_{\mu}$, and axial mediator, $A_{\mu}$, allowing us to cover the standard cases of spin-independent (SI) and spindependent (SD) scattering.

The interaction Lagrangian takes the form,

$$
\begin{aligned}
\mathcal{L}_{\text {int }} \supset & g_{\chi v} V_{\mu} \bar{\chi} \gamma^{\mu} \chi+g_{N v} V_{\mu} \bar{N} \gamma^{\mu} N \\
& +g_{\chi a} A_{\mu} \bar{\chi} \gamma^{\mu} \gamma^{5} \chi+g_{N a} A_{\mu} \bar{N} \gamma^{\mu} \gamma^{5} N
\end{aligned}
$$

where $\chi$ refers to the DM, and $N$ represents neutrons $(n)$ and protons $(p)$. We have also introduced the DM and nucleon couplings to the mediators $g_{\chi j}$ and $g_{N j}$, where $j=v, a$ denotes the vector and axial couplings, respectively. The dark matter can be treated as being at rest in the galaxy given the large CR energies. However, for heavier nuclei one must account for the momenta of the individual nucleons participating in the scattering process within the nucleus. For nonrelativistic dark matter-nucleus scattering one can apply the standard treatment of [39]. In this work we adopt the recent analysis of neutrino-nucleus scattering analyzed in [40], which closely resembles the physical situation of cosmic-ray scattered dark matter due to the relativistic nature of the incident neutrinos. This approach incorporates the momentum of the interacting nucleon, rather than treating it as being at rest, and also does not include contributions from nuclear states whose spin is flipped, which would not be present in the coherent scattering process. Following this avenue allows us to connect the nucleon cross-section to the full nuclear crosssection by relating the nucleon momenta to the full momentum transferred in the scattering process from energy-momentum conservation. This, for example, produces the propagator as a function of $m_{i}$ in the denominator rather than $m_{N}$ as the momentum is transferred to the full nucleus. Using this procedure we find that the vector and axial interactions generate the differential cross sections,

$$
\begin{aligned}
\left(\frac{d \sigma_{\chi T}}{d T_{\chi}}\right)_{\text {vector,CR }}= & g_{\chi v}^{2} g_{N v}^{2} A^{2} F^{2}\left(q^{2}\right) \\
& \times \frac{G\left(m_{\chi}, m_{N}, T_{i}, T_{\chi}\right)}{4 \pi\left(2 m_{\chi} T_{\chi}+m_{V}^{2}\right)^{2}\left(T_{i}^{2}+2 m_{i} T_{i}\right)}
\end{aligned}
$$

where $G\left(m_{\chi}, m_{N}, T_{i}, T_{\chi}\right) \equiv\left(m_{i} / m_{N}\right)^{2}\left(2 m_{\chi}\left(m_{N}+T_{i} / A\right)^{2}-\right.$ $\left.T_{\chi}\left(\left(m_{N}+m_{\chi}\right)^{2}+2 m_{\chi} T_{i} / A\right)+m_{\chi} T_{\chi}^{2}\right)$, and

$$
\begin{aligned}
\left(\frac{d \sigma_{\chi N}}{d T_{\chi}}\right)_{\text {axial,CR }}= & g_{\chi a}^{2} g_{N a}^{2} F^{2}\left(q^{2}\right) \\
& \times \frac{H\left(m_{\chi}, m_{N}, T_{i}, T_{\chi}\right)}{4 \pi\left(2 m_{\chi} T_{\chi}+m_{a}^{2}\right)^{2}\left(T_{i}^{2}+2 m_{N} T_{i}\right)}
\end{aligned}
$$

respectively, where $H\left(m_{\chi}, m_{N}, T_{i}, T_{\chi}\right) \equiv\left[2 m_{\chi}\left(2 m_{N}^{2}+\right.\right.$ $\left.\left.\left(m_{N}^{2}+T_{i}\right)^{2}\right)+T_{\chi}\left(\left(m_{N}+m_{\chi}\right)^{2}-2 m_{\chi} T_{i}\right)+m_{\chi} T_{\chi}^{2}\right]$. Here $A$ is the total nucleon number of the cosmic ray nucleus, and $m_{\chi}, m_{N}, m_{i}, m_{v}, m_{a}$ are the DM, nucleon, incident CR, vector and axial mediator masses, while $T_{\chi}$ is the outgoing DM kinetic energy. While the couplings may in principle be different, throughout this analysis we fix $g_{i} \equiv g_{\chi i}=g_{N i}$ where $i=V, A$ for simplicity. For the form factor, $F^{2}\left(q^{2}\right)$, we follow [15] and use the dipole form of [41] for protons but adopt the Helm model [42] for all larger nuclei (nuclei as light as the deuteron are reasonably well described by the Helm form factor [43]). In the case of the axial cross section, only proton scattering is considered and so the dipole form factor with the axial mass is sufficient [44].

In Fig. 1 we plot the total integrated cross section for $\mathrm{DM}$ incident on a nucleus at rest, for a $1 \mathrm{GeV}$ mediator and a variety of DM masses. The coupling choice $g=$ $\sqrt{\mathrm{MeV} / m_{\chi}}$ is chosen for illustration such that the cross sections converge to a common value in the nonrelativistic limit. As can be seen, there is a strong dependence on the DM mass, with light DM $\left(m_{\chi}<1 \mathrm{MeV}\right)$ masses the cross section grows substantially with increasing CR energy,

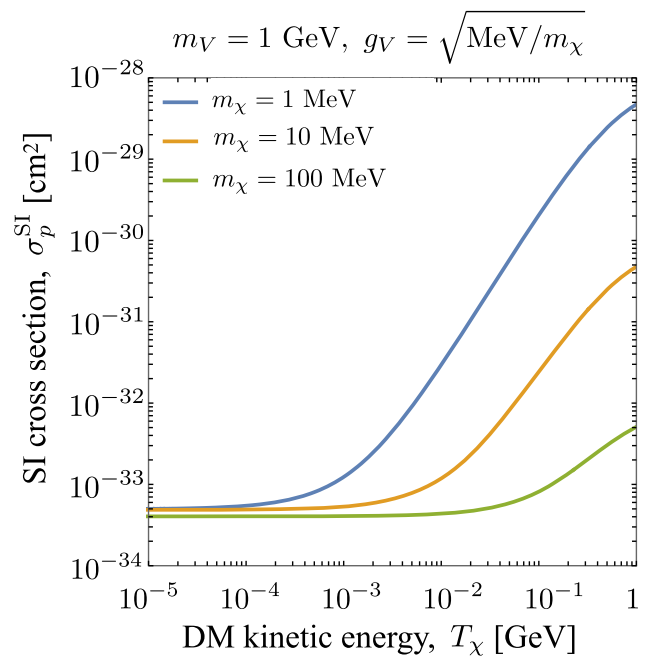

FIG. 1. Direct detection DM-nucleus cross section as a function of incoming DM kinetic energy for a variety of DM masses (for at rest nuclei). For illustration, the vector mediator mass is chosen to be $m_{V}=1 \mathrm{GeV}$. The coupling choice $g=\sqrt{\mathrm{MeV} / m_{\chi}}$ ensures that the cross sections converge to the same value in the nonrelativistic limit. 
while for heavier DM $\left(m_{\chi}>50 \mathrm{MeV}\right)$, the cross section drops precipitously.

This simple result motivates a careful reexamination of the sensitivity of direct detection experiments to this relativistic flux of DM.

\section{CALCULATIONAL FRAMEWORK}

Next we reformulate the cosmic ray dark matter spectrum retaining energy dependence of the cross section, which is required for even the simplest vector-vector interaction. The double-differential collision rate of CR particles $i$ with dark matter within an infinitesimal volume $d V$ is [15]

$$
\frac{d^{2} \Gamma_{\mathrm{CR}_{i} \rightarrow \chi}}{d T_{i} d T_{\chi}}=\frac{\rho_{\chi}}{m_{\chi}} \frac{d \sigma_{\chi i}}{d T_{\chi}} \frac{d \Phi_{i}^{\mathrm{LIS}}}{d T_{i}} d V
$$

The scattered dark matter flux is then obtained by integrating this over the relevant volume and cosmic ray energies

$$
\begin{aligned}
\frac{d \Phi_{\chi}}{d T_{\chi}} & =\int_{V} d V \int_{T_{i}^{\text {min }}} d T_{i} \frac{d^{2} \Gamma_{\mathrm{CR}_{i} \rightarrow \chi}}{d T_{i} d T_{\chi}} \\
& =D_{\text {eff }} \frac{\rho_{\chi}}{m_{\chi}} \sum_{i} \int_{T_{i}^{\min }} d T_{i} \frac{d \sigma_{\chi i}}{d T_{\chi}} \frac{d \Phi_{i}^{\mathrm{LIS}}}{d T_{i}}
\end{aligned}
$$

where $D_{\text {eff }}=1 \mathrm{kpc}$ is an effective diffusion zone parameter, $\rho_{\chi}=0.3 \mathrm{GeV} \mathrm{cm}^{-3}$ is the local DM density, $d \Phi_{i}^{\mathrm{LIS}} / d T_{i}$ is the local interstellar (LIS) flux of nuclear species $i$ [45], and $T_{i}^{\mathrm{min}}$ is the minimum incoming CR kinetic energy needed to produce an upscattered DM particle with kinetic energy $T_{\chi}, T_{i}^{\min }=\left(T_{\chi} / 2-m_{i}\right)$ $\left(1 \pm\left(1+2 T_{\chi}\left(m_{i}+m_{\chi}\right)^{2} /\left(m_{\chi}\left(2 m_{i}-T_{\chi}\right)^{2}\right)^{1 / 2}\right.\right.$. We follow Ref. [15] and include upscattering due to protons and helium, with the fluxes of CRs drawn from Ref. [45]. Note that the parameter $D_{\text {eff }}$ is essentially the distance over which the calculation accounts for CRs as the source of high-energy DM flux. While there is uncertainty in the precise value of this parameter, we follow Ref. [15] and conservatively set $D_{\text {eff }}=1 \mathrm{kpc}$ throughout.

Finally we compute the differential event rate (per unit detector mass) from the incoming relativistic DM flux in a direct detection experiment via

$$
\frac{d R}{d E_{T}}=\frac{1}{m_{\mathrm{T}}} \int_{T_{\chi}^{\min }}^{\infty} d T_{\chi} \frac{d \Phi_{\chi}}{d T_{\chi}} \frac{d \sigma_{\chi T}}{d E_{T}}
$$

where $E_{T}$ is the target recoil energy, and $T_{\chi}^{\min }$ can be found by taking the previously given expression for $T_{i}^{\text {min }}$ and making the substitutions $i \leftrightarrow \chi$, essentially reversing the roles of the incident and scattered particles. For vector and axial-vector exchange the total differential cross section $d \sigma_{\chi T} / d E_{T}$ can be obtained from Eqs. (2) and (3) through the substitutions $m_{\chi} \leftrightarrow m_{N}, T_{i} \rightarrow T_{\chi}, T_{\chi} \rightarrow E_{T}$, and the exchanged four-momentum will become $q^{2}=-2 m_{\mathrm{T}} E_{\mathrm{T}}$, where $m_{\mathrm{T}}$ is the target mass of the detector nuclei.

\section{ATTENUATION}

Most direct detection experiments are deep underground in order to suppress large backgrounds from activity at the surface. For example, XENON1T is operated at the Laboratori Nazionali del Gran Sasso (LNGS) situated at a depth of $z \simeq 1.4 \mathrm{~km}$. As a result of the Earth overburden, cross sections above some critical value will attenuate and decelerate the DM flux to such small energies that it is rendered undetectable (see e.g., Refs. [46-55] for studies of the attenuation of nonrelativistic DM).

As in Ref. [15], we evaluate the energy loss of dark matter of energy $T_{\chi}$ with respect to the distance $x$ traveled through the Earth. This depends on the energy $T_{r}$ lost by the dark matter in each collision through the relation

$$
\frac{d T_{\chi}}{d x}=-\sum_{T} n_{T} \int \frac{d \sigma}{d T_{r}} T_{r} d T_{r}
$$

where the sum is over the average nuclei densities $n_{T}$ of elements within the Earth. We use the values of $n_{T}$ provided by DARKSUSY [56].

While a detailed study of the effects of attenuation on CR-boosted DM is beyond the scope of this paper, we adopt the following prescription for determining the largest cross section underground experiments can probe. CRDM of a given mass will impinge upon the Earth with an initial energy, $T_{\chi, \text { in }}$. As the dark matter travels through the Earth, it will lose energy as described in Eq. (8) leaving it with a kinetic energy of $T_{\chi, z}$ once it reaches the depth, $z$, of XENON1T. For a given $T_{\chi, z}$, the maximal nuclear recoil within the detector is $E_{\mathrm{T}}=\frac{T_{\chi, z}^{2}+2 m_{\chi} T_{\chi, z}}{T_{\chi, z}+\left(m_{\chi}+m_{\mathrm{T}}\right)^{2} / 2 m_{\mathrm{T}}}$. Thus, there will be a maximal cross-section above which CRDM striking the Earth with an energy $T_{\chi, \text { in }}$, will be decelerated below the detector threshold energy $E_{\mathrm{th}}$. As a simple prescription we determine the upper bounds of the cross section exclusion plot by finding the cross sections above which an initial $T_{\chi}=1 \mathrm{GeV}$ would attenuate below threshold. We only consider the effects from elastic scattering, and the upper bounds may change if inelastic effects are included.

\section{RESULTS}

In Fig. 2 we present our results by computing constraints on SI and SD cross-sections within our simplified model framework, using XENON1T and Borexino data respectively. Here the coupling is fixed to $g=1$ and the mediator mass is varied as $\sigma=4 g^{4} \mu^{2} /\left(\pi m_{V}^{4}\right)$. One can see that the exclusion region now strongly depends on $m_{\chi}$, and in the vector case these bounds are extended by orders of 

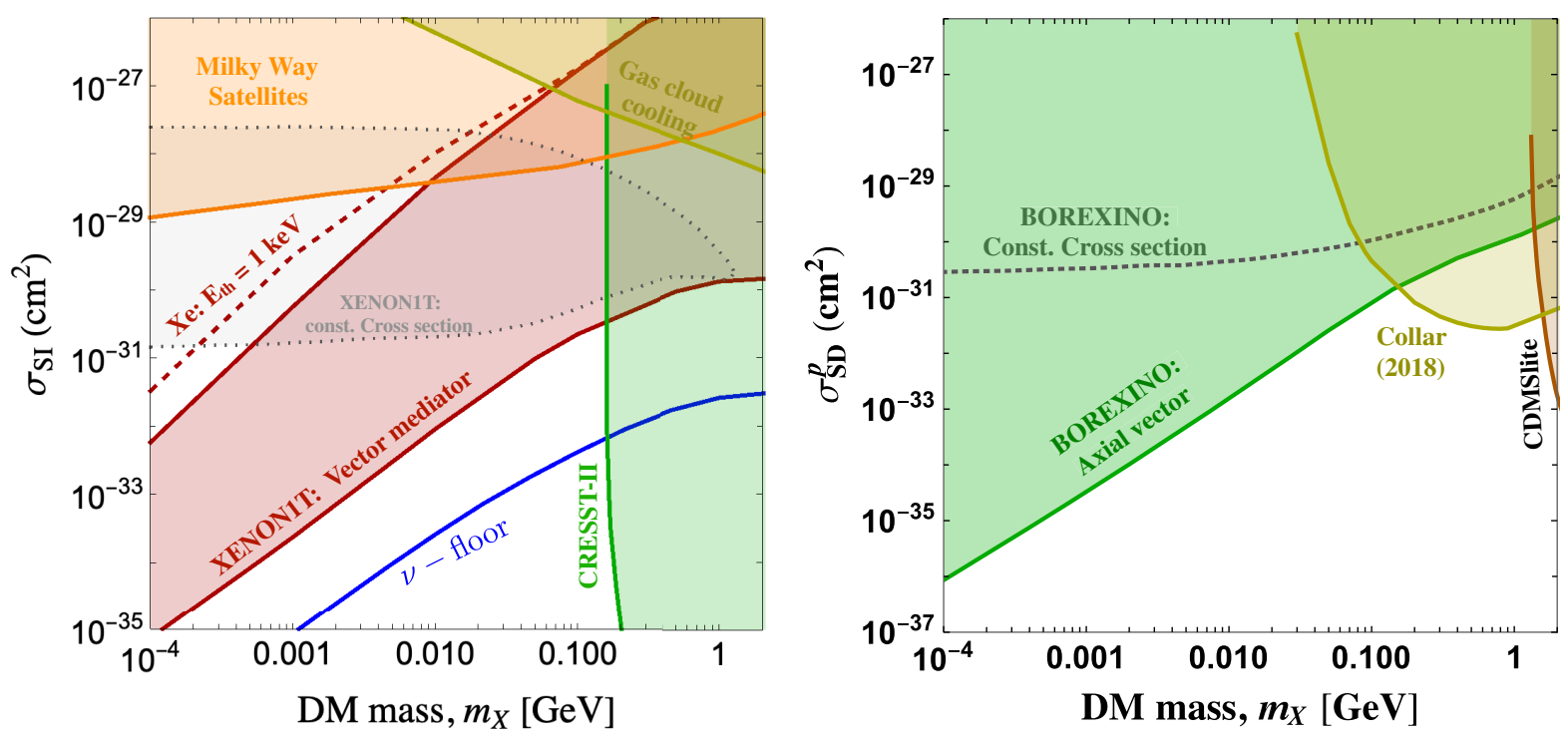

FIG. 2. Bounds on the SI (left) and SD (right) DM-proton cross sections. For vector interactions (SI) we examine XENON1T data ( vector case, the upper dashed line shows the sensitivity to dark matter attenuated by the Earth due to a lower detector threshold of $E_{\text {th }}=1 \mathrm{keV}$, approximately corresponding to the threshold of the XENON1T S2-only analysis [57]. For comparison, bounds from [15] which use an energy independent cross section are shown in dotted gray. We also display bounds from the conventional non-relativistic local DM. The additional bounds we display include gas cloud cooling [58], Milky Way satellites [59], above ground CRESST-II [8], CDMSlite [7], and Collar [60].

magnitude relative to an energy independent cross section as in Ref. [15]. We also note that the upper bound on the cross section displays a detector threshold dependence, emphasizing the enhanced reach of low threshold detection.

The neutrino background due to coherent elastic neutrino nucleus scattering (CE $\nu$ NS) originating from solar and atmospheric neutrinos will provide an irreducible background to CRDM scattering in liquid xenon experiments. Since the nuclear recoil spectrum of CRDM differs from the $\mathrm{CE} \nu \mathrm{NS}$ spectrum, there will not be a true "neutrino floor" in the sense of having a nearly identical spectral form [61]. To illustrate the sensitivity for which the neutrino background will start to become relevant, we calculate the cross section at which the total rate from CRDM is equal to the total rate of atmospheric neutrinos above $5 \mathrm{keV}$. The curve denotes the approximate point at which the experiment's sensitivity to CRDM is altered from depending on the inverse square root of exposure, to the inverse fourth-root. In the case of the SD scenario, Borexino is already sensitive to atmospheric neutrinos (see [62]) and thus can be considered to have already surpassed the floor.

We note that in Fig. 2, the constraints on the cross section values are quoted in the limit of no momentum transfer. This can obscure the bounds on the simplified model since there will be an interplay between the mediator mass and momentum transfer. Therefore, it can be insightful to examine bounds in the, $m_{V}$ vs $m_{\chi}$ plane, as shown in
Fig. 3. The coupling remains a free parameter and so we calculate the bounds for $g=1$. Larger couplings would enable bounds on larger mediator masses. Conversely, lighter mediators enhance the rate and enable bounds with smaller couplings, but when $m_{V}^{2}<q^{2}$ the rate will not be enhanced further and $g \sim 0.1$ is an approximate lower limit on the couplings accessible.

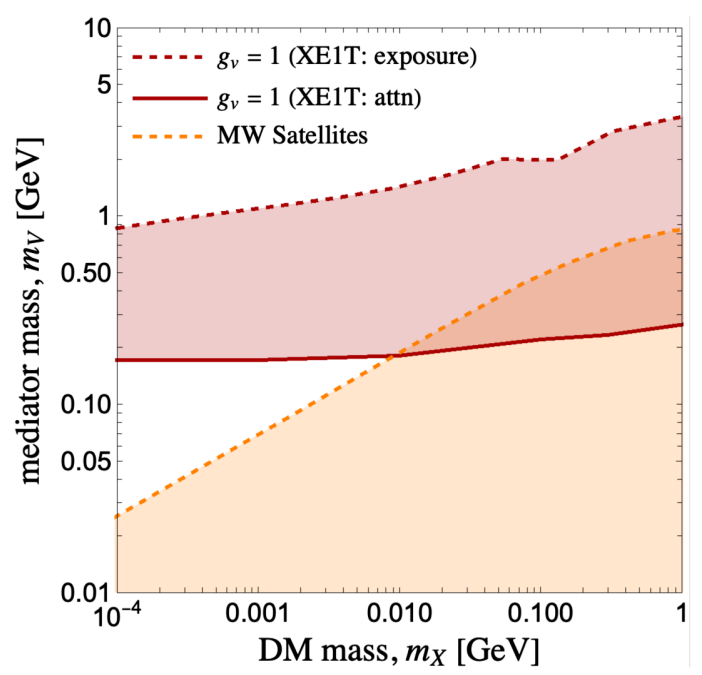

FIG. 3. Combination of bounds on the vector model with couplings set to unity. The red region shows the mediator and DM masses excluded by the search by XENON1T. The orange region is excluded by Milky Way satellites [59]. 
The shapes of the constraints in Fig. 2 can be explained using Fig. 3 roughly as follows. For the energy independent interactions of [15] the propagator depends solely on a fixed scale for the propagator with no $m_{\chi}$ or $T_{\chi}$ dependence, and the bounds are flat. However, in the present case the differential cross sections have a dependence from the propagator of $\left(2 m_{\chi} T_{\chi}+m_{V}^{2}\right)^{-2}$. As $m_{\chi}$ is varied, in order to maintain the same rate that is on the boundary of detectability, $m_{V}$ will also vary. For the sub-GeV $m_{\chi}$ range under consideration, $\mu \simeq m_{\chi}$, and, as can be seen from Fig. 3, as $m_{\chi}$ decreases from $1 \mathrm{GeV}$ down to $10^{-4} \mathrm{GeV}, m_{V}$ declines by about a factor of four. Thus, the ratio $m_{\chi}^{2} / m_{v}^{4}$, and therefore $\sigma$, has decreased by about $10^{-6}$ (modulo order one factors) over the same mass range.

In this paper we do not use any model but our results can be applied to a model where the constraints on the coupling constants may need to include the RG running [63]. As a UV completion of these models with light mediators, one can utilize the $U(1)_{T 3 R}$ gauge symmetry extensions of the SM [34] in order to have a concrete model of light mediators. In the context of a light DM model with light mediators, the CMB constraints need to be satisfied, which however, can be addressed by having the relic abundance emerging mostly from a $p$-wave dominated process [34] or from a DM particleantiparticle asymmetry.

\section{CONCLUSIONS}

In this paper we considered the impact of realistic energy dependence on the flux and scattering rate of CR-boosted DM. Our work suggests a number of avenues for future exploration. First, we have mostly focused on direct detection experiments, though as seen in the axial vector case, neutrino experiments can probe relevant parameter space, (see also [15,17]). These bounds also need to be revisited in light of the importance of energy dependent scattering. A more complete treatment would include hadronic inelastic processes, which may be relevant for the initial upscattering, DM attenuation, and detector energy deposition.

In addition, the energy dependence in realistic models explored here also modifies the bounds from Ref. [14] derived from cosmic ray energy losses, as well as those obtained in [17] for a simplified model approach to DMelectron scattering when DM-electron couplings are introduced. For purposes of illustration we have focused on CR-DM interactions mediated by the exchange of a (axial-) vector mediator. This is of course only one possible class of models, and we plan to follow-up on this paper with an exploration of additional interactions mediating SI and SD scattering including momentum dependent scattering. The bounds would be interesting for pseudoscalar models where the usual direct detection bounds are weak. Finally, due to the size of the energy transfer in the CRDM paradigm, it may be an intriguing space to explore inelastic dark matter models, as recently examined within boosted dark matter scenarios [64-67].

\section{ACKNOWLEDGMENTS}

We are grateful for helpful discussion with Chris Cappiello, Shunsaku Horiuchi, and Maxim Pospelov. B. D. acknowledges support from DOE Grant No. desc0010813. The work of I.M.S. is supported by the U.S. Department of Energy under the Award No. DE-SC0020250. J. L. N. is supported in part by the Australian Research Council. J. B. D. acknowledges support from the National Science Foundation under Grant No. NSF PHY-1820801. The research of J. B. D. was also supported in part by NSF Grant No. PHY-1748958.

\section{APPENDIX: MILKY WAY SATELLITE BOUND PROJECTION}

In this Appendix we discuss possible effects of momentum dependence of the Milky Way satellite bounds found in [59] on their projection in Fig. 2. We find that the maximum possible momentum transfer is negligible for the mediator masses under consideration.

The momentum transfer needs to be found at the redshift, $z$, where [59]

$$
a H=\left.R_{\chi}\right|_{z=z_{\text {crit }}}
$$

From Fig. 1 (left) of [25], we see this occurs at $z \gtrsim 10^{4}$. The temperature at this time is

$$
\begin{aligned}
T\left(z_{\text {crit }}\right)=T_{0}\left(1+z_{\text {crit }}\right) & =2.725\left(1+10^{4}\right) \frac{1 \mathrm{eV}}{11606 \mathrm{~K}} \\
& =2.34816 \mathrm{eV}
\end{aligned}
$$

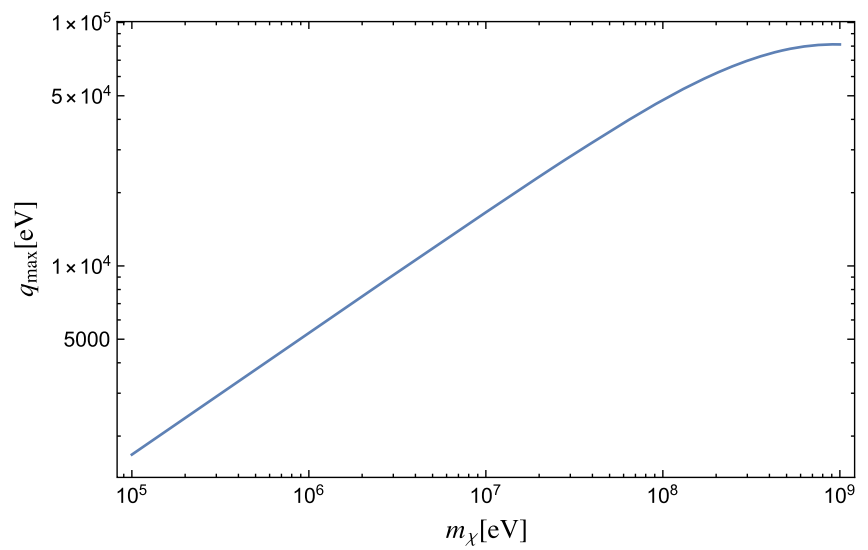

FIG. 4. The maximum value of momentum transfer as a function of dark matter mass evaluated at $z=10^{4}$. 
The dark matter temperature is actually less than this at $z=10^{4}$ (as seen in Fig. 1 (right) of [25]), meaning this is a conservative limit. The dark matter in the mass range of interest in the present work should be deep in the nonrelativistic regime at this time with a speed given by

$$
\frac{m_{\chi} v^{2}}{2}=\frac{3}{2} T \Rightarrow v=\sqrt{\frac{3 T}{m_{\chi}}}=\sqrt{\frac{3 T_{0}\left(1+z_{\text {crit }}\right)}{m_{\chi}}}
$$

The maximum momentum transfer in the interaction $\chi+p \rightarrow \chi+p$ is given in terms of the dark matter-proton reduced mass $\mu_{\chi p}$ as

$$
\left|\vec{q}_{\max }\right|=2 \mu_{\chi p} v .
$$

Using Eq. (A3) this becomes

$$
\left|\vec{q}_{\max }\right|=2 \mu_{\chi p} \sqrt{\frac{3 T_{0}\left(1+z_{\text {crit }}\right)}{m_{\chi}} .}
$$

In Fig. 4 we have plotted $\left|\vec{q}_{\max }\right|$ as a function of dark matter mass for the range $m_{\chi} \in\left[10^{5} \mathrm{eV}, 10^{9} \mathrm{eV}\right]$. The maximal value attained is $\left|\vec{q}_{\max }\right|=81.3 \mathrm{keV}$.

As seen in Fig. 3, the smallest mediator mass considered in this dark matter range is approximately $10 \mathrm{MeV}$, meaning that the momentum dependence will be negligible in the propagator term. The dipole form of the propagator contains a momentum dependent term that contains $Q^{2} / \Lambda^{2}$ where $\Lambda=770 \mathrm{MeV}$ for the proton, which will therefore create a minimal contribution at the maximum momentum value, and the Helm form factor will also produce a negligible effect with this momentum transfer.
[1] M. W. Goodman and E. Witten, Phys. Rev. D 31, 3059 (1985).

[2] Z. Z. Liu et al. (CDEX Collaboration), Phys. Rev. Lett. 123, 161301 (2019).

[3] X. Ren et al. (PandaX-II Collaboration), Phys. Rev. Lett. 121, 021304 (2018).

[4] P. Agnes et al. (DarkSide Collaboration), Phys. Rev. Lett. 121, 081307 (2018).

[5] D. S. Akerib et al. (LUX Collaboration), Phys. Rev. Lett. 118, 251302 (2017).

[6] R. Agnese et al. (SuperCDMS Collaboration), Phys. Rev. Lett. 120, 061802 (2018).

[7] R. Agnese et al. (SuperCDMS Collaboration), Phys. Rev. D 97, 022002 (2018).

[8] G. Angloher et al. (CRESST Collaboration), Eur. Phys. J. C 77, 637 (2017).

[9] F. Petricca et al. (CRESST Collaboration), J. Phys. Conf. Ser. 1342, 012076 (2019).

[10] A. Aguilar-Arevalo et al. (DAMIC Collaboration), Phys. Rev. D 94, 082006 (2016).

[11] C. Amole et al. (PICO Collaboration), Phys. Rev. Lett. 118, 251301 (2017).

[12] R. Agnese et al. (SuperCDMS Collaboration), Phys. Rev. Lett. 121, 051301 (2018); 122, 069901(E) (2019).

[13] M. Crisler, R. Essig, J. Estrada, G. Fernandez, J. Tiffenberg, M. Sofo haro, T. Volansky, and T.-T. Yu (SENSEI Collaboration), Phys. Rev. Lett. 121, 061803 (2018).

[14] C. V. Cappiello, K. C. Y. Ng, and J. F. Beacom, Phys. Rev. D 99, 063004 (2019).

[15] T. Bringmann and M. Pospelov, Phys. Rev. Lett. 122, 171801 (2019).

[16] C. Cappiello and J. F. Beacom, Phys. Rev. D 100, 103011 (2019).

[17] Y. Ema, F. Sala, and R. Sato, Phys. Rev. Lett. 122, 181802 (2019).
[18] M. Ibe, W. Nakano, Y. Shoji, and K. Suzuki, J. High Energy Phys. 03 (2018) 194.

[19] M. J. Dolan, F. Kahlhoefer, and C. McCabe, Phys. Rev. Lett. 121, 101801 (2018).

[20] D. S. Akerib et al. (LUX Collaboration), Phys. Rev. Lett. 122, 131301 (2019).

[21] E. Armengaud et al. (EDELWEISS Collaboration), Phys. Rev. D 99, 082003 (2019).

[22] N. F. Bell, J. B. Dent, J. L. Newstead, S. Sabharwale, and T. J. Weiler, Phys. Rev. D 101, 015012 (2020).

[23] C. Kouvaris and J. Pradler, Phys. Rev. Lett. 118, 031803 (2017).

[24] V. Gluscevic and K. K. Boddy, Phys. Rev. Lett. 121, 081301 (2018).

[25] K. K. Boddy and V. Gluscevic, Phys. Rev. D 98, 083510 (2018).

[26] K. K. Boddy, V. Gluscevic, V. Poulin, E. D. Kovetz, M. Kamionkowski, and R. Barkana, Phys. Rev. D 98, 123506 (2018).

[27] J. Alvey, M. Campos, M. Fairbairn, and T. You, Phys. Rev. Lett. 123, 261802 (2019).

[28] J. F. Cherry, M. T. Frandsen, and I. M. Shoemaker, Phys. Rev. Lett. 114, 231303 (2015).

[29] M. Pospelov, A. Ritz, and M. B. Voloshin, Phys. Lett. B 662, 53 (2008).

[30] D. Hooper and K. M. Zurek, Phys. Rev. D 77, 087302 (2008).

[31] C. Cheung, J. T. Ruderman, L.-T. Wang, and I. Yavin, Phys. Rev. D 80, 035008 (2009).

[32] R. Essig, J. Kaplan, P. Schuster, and N. Toro, arXiv:1004 .0691.

[33] R. Essig et al., in Proceedings, 2013 Community Summer Study on the Future of U.S. Particle Physics: Snowmass on the Mississippi (CSS2013): Minneapolis, MN, USA (2013) [arXiv:1311.0029]. 
[34] B. Dutta, S. Ghosh, and J. Kumar, Phys. Rev. D 100, 075028 (2019).

[35] O. Buchmueller, M. J. Dolan, S. A. Malik, and C. McCabe, J. High Energy Phys. 01 (2015) 037.

[36] J. Abdallah et al., Phys. Dark Universe 9-10, 8 (2015).

[37] A. Berlin, D. Hooper, and S. D. McDermott, Phys. Rev. D 89, 115022 (2014).

[38] J. B. Dent, L. M. Krauss, J. L. Newstead, and S. Sabharwal, Phys. Rev. D 92, 063515 (2015).

[39] N. Anand, A. L. Fitzpatrick, and W. C. Haxton, Phys. Rev. C 89, 065501 (2014).

[40] V. A. Bednyakov and D. V. Naumov, Phys. Rev. D 98, 053004 (2018).

[41] C. F. Perdrisat, V. Punjabi, and M. Vanderhaeghen, Prog. Part. Nucl. Phys. 59, 694 (2007).

[42] R. H. Helm, Phys. Rev. 104, 1466 (1956).

[43] C. Krber, A. Nogga, and J. de Vries, Phys. Rev. C 96, 035805 (2017).

[44] B. Bhattacharya, G. Paz, and A. J. Tropiano, Phys. Rev. D 92, 113011 (2015).

[45] D. Bisschoff, M. S. Potgieter, and O. P. M. Aslam, Astrophys. J. 878, 59 (2019).

[46] J. I. Collar and F. T. Avignone III, Phys. Rev. D 47, 5238 (1993).

[47] F. Hasenbalg, D. Abriola, F. T. Avignone, J. I. Collar, D. E. Di Gregorio, A. O. Gattone, H. Huck, D. Tomasi, and I. Urteaga, Phys. Rev. D 55, 7350 (1997).

[48] G. Zaharijas and G. R. Farrar, Phys. Rev. D 72, 083502 (2005).

[49] G. D. Mack, J. F. Beacom, and G. Bertone, Phys. Rev. D 76, 043523 (2007).

[50] R. Foot, Phys. Rev. D 69, 036001 (2004).

[51] C. Kouvaris and I. M. Shoemaker, Phys. Rev. D 90, 095011 (2014).
[52] B. J. Kavanagh, R. Catena, and C. Kouvaris, J. Cosmol. Astropart. Phys. 01 (2017) 012.

[53] T. Emken and C. Kouvaris, J. Cosmol. Astropart. Phys. 10 (2017) 031.

[54] T. Emken and C. Kouvaris, Phys. Rev. D 97, 115047 (2018).

[55] J. Bramante, B. Broerman, R. F. Lang, and N. Raj, Phys. Rev. D 98, 083516 (2018).

[56] T. Bringmann, J. Edsj, P. Gondolo, P. Ullio, and L. Bergstrm, J. Cosmol. Astropart. Phys. 07 (2018) 033.

[57] E. Aprile et al. (XENON Collaboration), Phys. Rev. Lett. 123, 251801 (2019).

[58] A. Bhoonah, J. Bramante, F. Elahi, and S. Schon, Phys. Rev. Lett. 121, 131101 (2018).

[59] E. O. Nadler, V. Gluscevic, K. K. Boddy, and R. H. Wechsler, Astrophys. J. 878, L32 (2019); Astrophys. J. Lett. 878, L32 (2019).

[60] J. I. Collar, Phys. Rev. D 98, 023005 (2018).

[61] J. B. Dent, B. Dutta, J. L. Newstead, and L. E. Strigari, Phys. Rev. D 93, 075018 (2016).

[62] V. S. Atroshchenko and E. A. Litvinovich, J. Phys. Conf. Ser. 675, 012014 (2016).

[63] F. D'Eramo, B. J. Kavanagh, and P. Panci, J. High Energy Phys. 08 (2016) 111.

[64] D. Tucker-Smith and N. Weiner, Phys. Rev. D 64, 043502 (2001).

[65] D. Kim, J.-C. Park, and S. Shin, Phys. Rev. Lett. 119, 161801 (2017).

[66] G. F. Giudice, D. Kim, J.-C. Park, and S. Shin, Phys. Lett. B 780, 543 (2018).

[67] C. Ha et al. (COSINE-100 Collaboration), Phys. Rev. Lett. 122, 131802 (2019). 